\title{
Energy Efficient Multipath Routing Protocol with Guaranteed Data Delivery for Wireless Sensor Network
}

\author{
Kirankumar B. Balavalad, Ajayakumar C. Katageri, Poornima M. Chanal, and Gururaj Kori
}

\begin{abstract}
The current challenge in wireless sensor networks is high data delivery ratio with low energy consumption. In this paper, a node that has collected maximum amount of data (buffer is maximum) is given first priority to access the channel, and send the collected data to the Sink through greedy routing. To achieve high delivery ratio with low energy consumption, data is forwarded along a multiple path to save energy, and a high data delivery ratio is achieved by path repair whenever a break is detected.We propose a simple path repairing protocol, whereby a node can skip over path break. Some of the important parameter like packet delivery ratio, path set up time and data transmission time; are studied.
\end{abstract}

Index Terms-Wireless sensor networks, energy efficient routing, guaranteed data delivery.

\section{INTRODUCTION}

A wireless sensor network consists of a large number of tiny sensing devices, deployed in a region of interest. Each device has processing and wireless communication capabilities, which enable it to gather information from the environment and to generate and deliver report messages to the remote base station (or sink node). The base station aggregates and analyzes the report messages received and decides whether there is an unusual or concerned event occurrence in the area of interest. The main challenge of wireless sensor network routing protocols is to achieve maximal robustness against path failure with minimal energy consumption.

In single-path routing, for each data packet, there is only one copy travelling along one path in the network. While in multi-path routing, multiple copies of one packet are transmitted in parallel along different paths to the same destination. Existing major routing protocols for wireless sensor networks include LEACH [1], Directed Diffusion [2], Energy Aware Routing [3], Rumor [4], Braided [5], and MESH etc.

Among the above-mentioned multi-hop protocols, only MESH is explicitly claimed as multi-path routing.

Braided builds multiple paths for a data delivery, but only one of them is used, while others are maintained as backup paths. Directed Diffusion can be single-path or multi-path routing depending on how many paths are reinforced by sink node. Energy aware routing and Rumor routing are single-path ones. Generally, single path routing is simple and

Manuscript received September 19, 2013; revised December 17, 2013.

The authors are with Basaveshwar Engineering College, Bagalkot Karnataka, 587 102, India (e-mail: kiranb4004@gmail.com, ajaykatageri@yahoo.co.in, poornima.chanal@yahoo.com, korigurus@yahoo.com). consumes less energy than multi-path routing. However, a single path failure will cause a break of transmission and hence completely destroy the delivery. Consequently, more and more researchers are resorting to multi-path routing for delivery success. For instance, sending the same data packet along two fully node-disjointed paths (if they exist) almost doubles the delivery ratio. Using $\mathrm{n}$-fully node-disjointed paths $(n>2)$ can further increase the delivery ratio in approximate proportion to $\mathrm{n}$. Moreover, if we relax the requirement for disjointed-ness, partial or interwoven multi-path routing schemes have shown higher resilience to single path failure theoretically and experimentally [6]. However, determining the width of multi-path routing (for instance, the value of $\mathrm{n}$ in $\mathrm{n}$-fully node-disjointed paths) before transmission is not so easy, because sensor network topologies often change unpredictably due to sudden node malfunction, environmental physical damage and impulsive strong external interference. Large ' $n$ ' values can ensure success of deliveries, but may cause unnecessary energy waste. In contrast, a small ' $n$ ' value saves energy, but may not guarantee the highly demanded delivery ratio. Another disadvantage of a large ' $n$ ' value is that: the larger the 'n' value is, the more traffic is generated for one data packet delivery, which may cause network congestion. Given that the simplest CSMA scheme is used at the MAC layer, more traffic means a longer back off delay waiting for transmission and more collisions induced in the wireless channel. Unless the source nodes are notified of path quality in a certain way, it is impossible to adjust the optimal $\mathrm{k}$ value dynamically to adapt to unpredictable network topology changes.

The rest of this paper is organized as follows: in Section II, current work in the area of energy efficient multipath routing protocol with guaranteed data delivery for WSN is reviewed. In Section III, the principle of the proposed protocol for WSN is explained in detail. Section IV, proposed model for WSN, Section V, evaluates the protocol through analysis and simulations. Section VI gives results and discussions. Finally, Section VII concludes the whole work. And Section VIII gives the references.

\section{RELATED WORKS}

The important issue to be considered from the communications point of view is routing. A good survey of major routing techniques in WSN is provided in [7]. The numerous challenges and issues that the developer must face while designing routing protocols for the transmission of data. There are many goals of Multipath routing protocols to achieve such as Reliability, Load Balancing, High Aggregate Bandwidth, Minimum End to End Delay, Minimum Energy consumption, High throughput. Ref. [8] Geographical 
Adaptive Fidelity (GAF) is introduced to reduce energy consumption in ad hoc wireless networks. GAF identifies equivalent nodes for routing based on location information and turns off unnecessary nodes. Yu et al. [9] has proposed a GEAR (Geographic Energy Aware Routing) algorithm which uses energy levels and geographic information of neighbours for selection and route the packet towards the target region. In GEAR, each node has an estimated cost and learning cost of reaching the destination through its neighbours. The estimated cost is the combination of residual energy and distance to destination. Ref. [10] Gradient-Based Routing: Schurgers et al. have proposed a slightly changed version of Directed Diffusion, called Gradient-based routing (GBR). The idea is to keep the number of hops when the interest is diffused through the network. Hence, each node can discover the minimum number of hops to the sink, which is called height of the node. The difference between a node's height and that of its neighbor is considered the gradient on that link. A packet is forwarded on a link with the largest gradient. The authors aim at using some auxiliary techniques such as data aggregation and traffic spreading along with GBR in order to balance the traffic uniformly over the network. Nodes acting as a relay for multiple paths can create a data combining entity in order to aggregate data. Ref. [11] the author proposes an energy efficient and collision aware (EECA) node-disjoint multipath routing algorithm for wireless sensor networks. With the aid of node position information, the EECA algorithm attempts to find two collision-free routes using constrained and power adjusted flooding and then transmits the data with minimum power needed through power control component of the protocol. Ref. [12] Power-Efficient Gathering in Sensor Information Systems (PEGASIS) is the protocol in which only one node is chosen as a head node that sends the fused data to the Base Station per round. PEGASIS protocol requires the formation of a chain, which is achieved in two steps. During the chain construction phase, the farthest node from the Base Station is considered first, and a greedy approach is followed to construct the chain. During the data gathering phase, a leader of each round is selected randomly. When a node dies, the chain is reconstructed to bypass the dead node. After the leader is selected, it passes the token to initiate the data gathering process. Passing the token also requires energy consumption but the cost of passing the token is very small because the token size is very small.

\section{PROPOSED WORK}

The method used for achieving a high delivery ratio for small ' $n$ ' value with low energy consumption is to forward data along a multi path (small ' $n$ ' value). The multi path routing table is constructed by comparing energy level of the inter-mediate node (greedy routing algorithm) with the threshold energy level. If the node has energy more than threshold it is selected for data transmission. Data will forward along these multi path and to repair the path whenever a break is detected. Path repair has been introduced into many wireless routing protocols [13], [14]. Depending on how the original path is established and what is the reason of path break, repair approaches are different. In [13], [14], when a path break (failure) is detected, a notification is sent to the source node, which is responsible for finding an alternative path and resending the data packet. However, this kind of source initiated path repairing approach is uneconomical, especially when a failure occurs many hops away from the source node.

In this work, we propose a local path repairing approach, whereby the node, which is located at the immediate upstream of a path break, is responsible for seeking alternative paths through a local survey. If alternative paths exist, data forwarding will proceed along the best of them without restarting from the source node. Although the selected alternative path may not be optimal from the view of the source node, the energy is conserved by preventing the previous transmission effort from being wasted, avoiding long-distance failure notification, and restricting the range of alternative path seeking into a small local area. Such energy saving should outweigh the additional energy caused by using a non optimal path.

If alternative path does not exit, data forwarding will proceed by finding the path which is less loaded among remaining multipath routes.

\section{PROPOSED MODEL}

Certain assumptions are made to design the proposed protocol.

1) All the nodes need not know topology of the network and, synchronization amongst all nodes is not required.

2) Each node is given a node ID and only has the addresses of the nodes that come under its range.

3) WSN consists of several sensor nodes deployed randomly in a region with a single sink.

4) Source node use multipath routing schemes to distribute the traffic.

This chapter explains the concept of proposed Energy Efficient Routing protocol with a Guaranteed Delivery with an example

\section{A. Network Environment}

A Wireless Sensor Network consisting of $N$ nodes is generated by random placement of the nodes within the given area. Each of these nodes has the fixed communication range and has fixed buffer and energy availability. All the nodes are static. Fixed sink is placed at one of the corners of the network. All the sensor nodes are aware of the sink position and the distance between itself and the sink. Multi-hop routing is considered.

\section{B. Energy Efficient Routing with Guaranteed Delivery Protocol}

In this proposed Model, buffer is considered to be one of the important parameter for media access. Buffer is used for storing the data that has been sensed. Sensor nodes will have to act as routers, and will have to receive packets from its neighbors to forward to the sink due to multi-hop nature of sensor networks. This traffic will increase the delay variation that packets incur while traveling on different routes and even on the same route.

Hence, the mechanism employed for energy efficient routing with guaranteed data delivery in proposed protocol is 
mainly based on the energy level and amount of buffer occupied at each sensor nodes. Whenever a node has collected important data to be transmitted, it broadcast the buffer size, i.e., amount of occupied buffer along with its node id, which is considered as beacon bit. Similarly all other nodes which have detected the event transmit a beacon. These nodes have now entered the competition. The nodes under competition will compare the buffer sizes and its energy level to the threshold energy level. if their buffer size is less than others, they come out of competition, i.e., the node with higher data to be sent, will have access to the channel. This is denoted as active node.

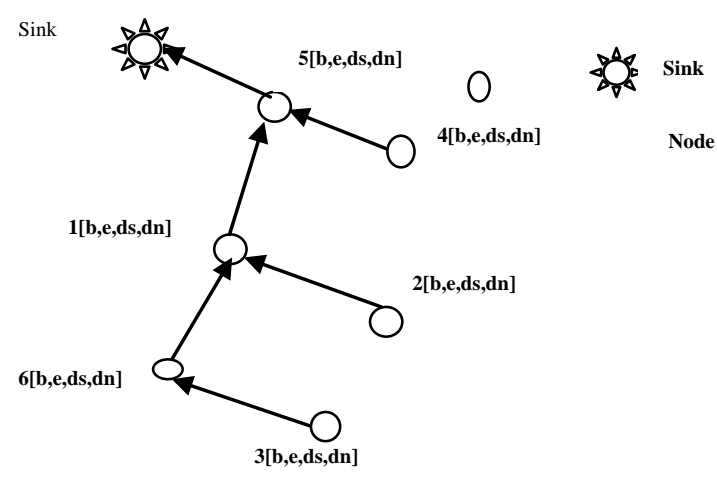

Fig. 1. Media access.

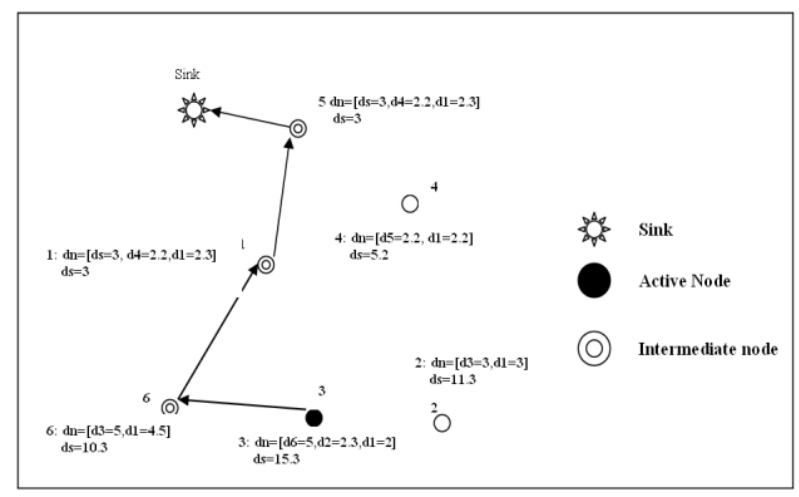

Fig. 2. Routing from node 3.

Fig. 1 shows three active nodes: 1, 3, and 5 with their respective occupied buffers and energy level. Neighboring nodes for 1 are 3, 4, 5, and 6. Neighboring nodes for 3 are 1, 2, and 6. Neighboring nodes for 5 are 1, 5, and sink. These nodes send beacon to their neighboring nodes as shown in the figure. In the above example, the nodes which are not active remain quite. Amongst these three nodes, node 3 has highest buffer and hence it is given chance to access the media.

For the purpose of routing, intermediate nodes are selected by comparing their energy level with threshold energy, if the node has maximum energy it is selected for routing. For routing, each node will have the addresses and distances of the sink and their neighboring nodes. Packets are marked by their originators with their destination's locations. As a result, a forwarding node can make a locally optimal, greedy choice in choosing a packet's next hop. Specifically, if a node knows its radio neighbor's positions, the locally optimal choice of next hop is the neighbor geographically closest to the packet's destination. Forwarding in this regime follows successively closer geographic hops, until the destination is reached. Thus form the path list of the nodes to be followed. Fig. 2 shows the routing from node 3 . For node 3 , the intermediate node is 6 , as it is having maximum energy and it is in range to 3 and it is nearest to sink and farthest from itself. Following the same method, node 6 finds node 1 as its next node, and node 1 finds node 5 as its next node, and node 5 is having sink in its range, and finally the sink is reached by following the path as [ 3 to 6 to 1 to 5 to sink]. This is the setup of path list to be followed by the active node.

This proposed work supports participation of multiple numbers of nodes for data transmission at a time if they are far away, or non-interfering with each. Suppose there are ' $n$ ' nodes in a given part ' $Y 1$ ' of the whole area ' $Y$ ' and ' $\mathrm{X}$ ' out of ' $\mathrm{n}$ ' are interested in transmitting after event detection. Applying the above theory of media access, out of ' $x$ ' $a$ single node will be selected as active node. In this way at a different corner part 'Y2' of the given area one more active node will be selected. And applying the same, multiple active nodes can be generated and all these active nodes will participate in transmission.

All these active nodes will find their path list. The nodes in the path list are intermediate nodes. Initially these will be in sleep mode. Once they get into the path list, the active nodes will send a flag bit to wake (switch $\mathrm{ON}$ ) them up. Technique which uses multiple radios, a low-power radio that is used exclusively to wake up the high power radio can also be used to wake these nodes. Buffer in these nodes may be used for self and are used for routing also.

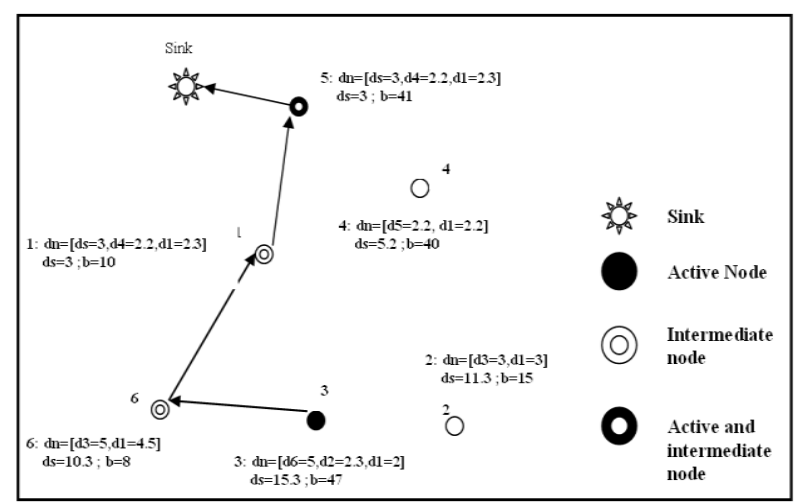

Fig. 3. Routing from active nodes

Fig. 3 shows routing. Active nodes are node 3 and node 5 . Both of the nodes are out of range from each other and can participate in data transmission at same time. Node 5 is directly reachable to sink and transmits data directly. Node 3 finds the path as 3 to 6 to 1 to 5 to sink and transmits accordingly.

Suppose if node 3 is transmitting and is on its path at node 1 and is ready to hop at node 5 . At the same time, node 5 may also get ready for data transmission. In such case, as node 5 has less buffer [ $b=41, E=45]$, as compared to node 3 [ $b=47$, $E=34]$, node 5 waits and allows node 3 to transmit data. After node 3's transmission is over, node 5 starts its data transmission.

\section{Simulation}

We have simulated the proposed model for a fixed area and random topology. The algorithm is executed to compute the different performance parameters. In this chapter, we 
discuss the simulation model and procedure used to test the proposed scheme.

\section{A. Simulation Model}

The proposed model has been simulated in various network scenarios on a Pentium-4 machine by using $\mathrm{C}$ programming language for the performance and effectiveness of the approach. Simulated Wireless Sensor Network area is considered as $\mathrm{A} \times \mathrm{B}$ sq meters. ' $\mathrm{N}$ ' number of sensor nodes are considered for event detection and one destination node is considered as Sink node for which all the other sensor nodes send the collected information. Out of total ' $N$ ' number of nodes, ' $n$ ' nodes or all nodes may become 'active', i.e., collect the information. The nodes are active with probability ' $\mathrm{P}$ '. ' $\mathrm{r}$ ' meters is transmission range is fixed for all nodes in the network. "Multiple sources to Single Sink" communication model is been considered and the performance parameters are analyzed. Buffer size is been equally fixed at each node. Buffer size at each node is ' $\mathrm{B}$ ' bytes. Each nodes are possess varying Energy level 'E' Joules. At each event detection, the buffer size occupied at the active nodes will vary. The network is static. The nodes which are present in the path traced are called intermediate nodes.

\section{B. Simulation Inputs}

To illustrate some results of the proposed WSN simulation, we have taken: Area of $40 \times 40$ sq.mts. Fixed network. Number of nodes is $N=10$ to 100 . Transmission range is same for all the nodes='r' meters. $B=60$. Energy $=E \mathrm{~J}$. At event detection, the buffer size occupied (utilized) varies from [0 to 40]. Probability (number of nodes active out of ' $N$ ' nodes) $P=0.25,0.5,0.75,1$. Sink is fixed.

\section{Simulation Procedure}

In this section, we briefly outline the simulation procedure of the proposed system.

\section{Begin}

- Generate the random network topology for a given number of nodes.

- Generate amount of utilized buffer randomly at each node.

- Activate some nodes considering them to have detected events. Amount of data detected is randomly generated.

- Compare the energy level of the active node and intermediate nodes with threshold energy level.

- Apply the proposed model for different parameter scenarios.

- Compute the performance of the system. End.

\section{Performance Parameters}

The performance parameters measured in the model are as follows:

- Path setup time: It is the time required to build the routing table, find the path between the active nodes and the Sink by considering the program execution in simulation.

- Data transmission time: It is time required to transmit packet over the multiple paths.

- Overall packet loss: Total sum of packets lost, considering packets getting transmitted from all active nodes towards the Sink. That is, at each path from active node to sink, some packets will be lost. Sum of these packets considering at all the active nodes is defined as overall packet loss.

\section{RESULTS AND DISCUSSIONS}

From Fig. 4, it is observed that, path setup time increases with increase in total number of nodes ' $\mathrm{N}$ ' and also as the transmission range of nodes is increased, Path setup time increases. Path setup time depends on three factors: execution time of the simulation, total number of nodes, and number of active nodes. Path setup time increases with number of nodes: as the number of nodes increases, the entries in the routing table (consisting of nodes along with its neighboring list, with respective buffer sizes and distances) also increases. Therefore the time for finding path (Greedy Routing Algorithm) increases. Hence path setup time depends on execution timing, number of nodes entries in the routing table, number of sources (active nodes). Also as the transmission range of the nodes is increased, this also results in addition of more number of neighbors in the neighboring list of the routing table. Increase in transmission range as $3 \mathrm{mts}, 5 \mathrm{mts}, 8 \mathrm{mts}$ shows increase in the path setup time.

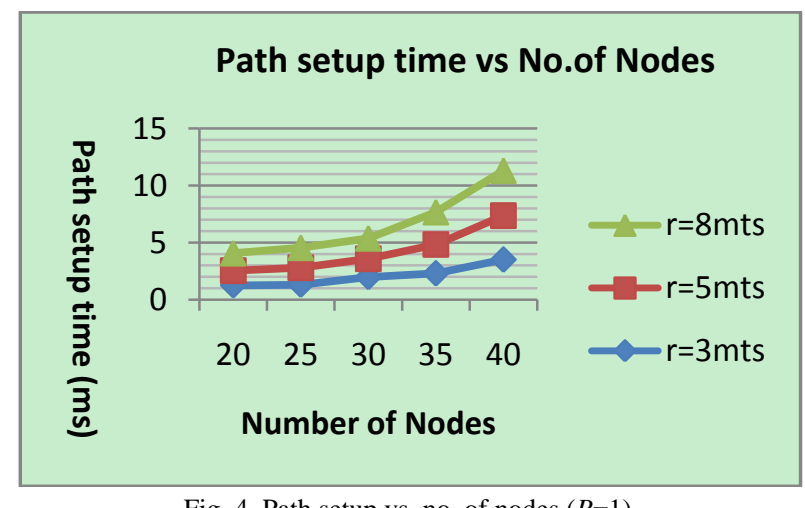

Fig. 4. Path setup vs. no. of nodes $(P=1)$.

Fig. 5 shows that as the probability of number of active nodes increases, Path setup time increases and also as the Transmission range of number of nodes increases, Path setup time increases. Increase in the probability implies increase in the number of active nodes. And for each active node path is to found. And the total path setup time is the addition of time spent at each active node. So the path setup time increases.

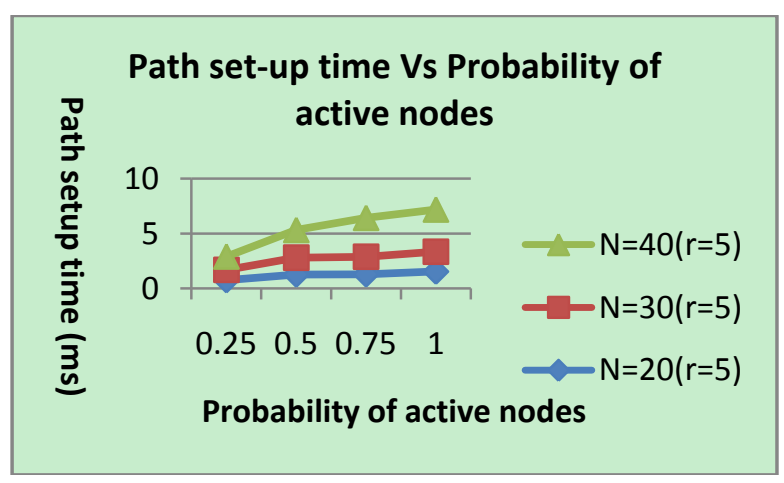

Fig. 5. Path setup time vs. probability of active nodes.

Fig. 6 shows that as the number of packets to be sent at the source node increases, (greater than free buffer) overall packet loss also increases. As the $\mathrm{P}$ is increased, traffic generated will be more, resulting in more packet loss. 


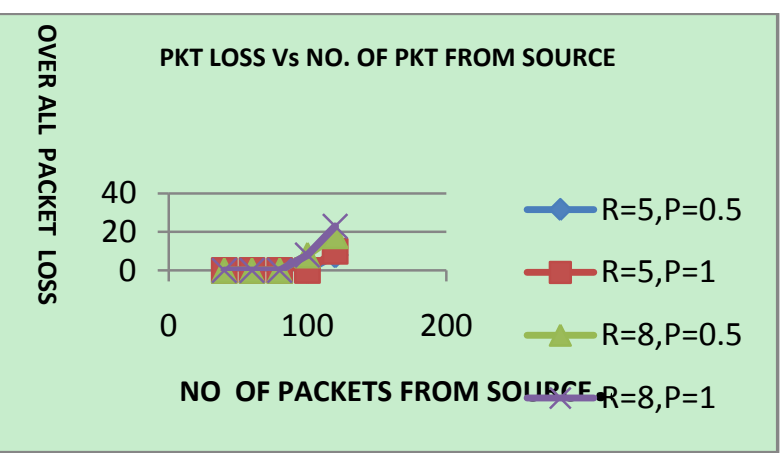

Fig. 6. Packet loss vs. number of packets from source nodes $(N=20)$.

In this protocol, Packet delivery ratio is totally dependent on the availability of buffer at the intermediate nodes and Sink. In this simulation model total Buffer $=60$ and free buffer $=30$. It is very clear from the graph that, till the packets to be transmitted are less than 40, packets lost are very less. This is because; the free buffer at all the nodes is fixed as 30 and total buffer is 60 . Slight linearity can be observed here. But as the number of packets is increased above 60, more packet loss can be seen. Hence in order to obtain more throughputs, the buffer size at the nodes can be increased.

Fig. 7 shows the Data transmission time Versus the Number of paths with varying data size. The packets are sent simultaneously sent over the multiple paths, therefore as there is increase in the number of paths the amount of time required to transmit the data is also decreased. The simulations are carried for varying data size.

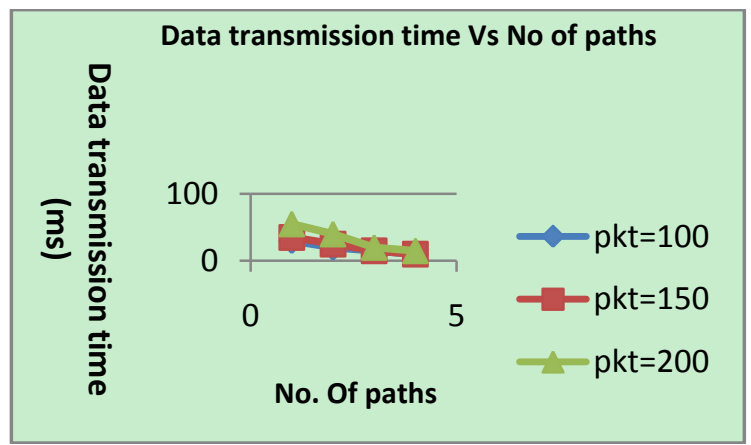

Fig. 7. Data transmission time vs no of paths.

\section{CONCLUSIONS AND EXTENSIONS}

In this paper, we focus on reliable data delivery scheme in wireless sensor networks. We design a multi-path routing with repair is an effective solution, which can simultaneously guarantee delivery and avoid energy waste. We propose a path repairing approach, which can quickly find an alternate path against a broken link by doing a small survey around the break and only using already existing routing information.

Special provisions in the protocol to handle a burst in the traffic is been done, by considering multiple-source to single sink. The protocol, as such, would survive although the performance could degrade to some extent, when all the nodes would detect and report at a time.

\section{A. Future Extensions for This Work Are}

\section{1) Large scale deployment}

In this proposed work it is seen that, as the number of nodes keep on increasing, the protocol work degrades. Hence extension in the project so as to handle large scale deployments is to be done as: Sensor networks will grow in size because of lower cost.

\section{2) Heterogeneous sensors}

Homogeneous network of sensors has been the typical assumption, but not the future!!

Combining sensors with different functions, hierarchy of sensors-a few expensive powerful sensors with more cheap sensors, for useful for special communication nodes, a few sensor nodes with expensive sensors, such as GPS-equipped sensors will have to used. These heterogeneous nodes will have different characteristics. Hence the protocol which can handle such heterogeneous nodes in a network is to be developed based on the platform of the presented work.

\section{3) Mobile sensors}

Most of the protocols work, which is been done in the field of WSN's is for non-mobile sensor nodes. The proposed work can be upgraded to handle mobile nodes by continuously updating the routing table. This extension would not be complicated, as already, it updates the routing table after the power loss and death of some sensor nodes. Extra task to be carried is to update at every movement of the sensor nodes. Low-Power Motors can Support Mobility for the sensors.

\section{REFERENCES}

[1] W. R. Heizelman, A. Chandrakasan, and H. Balakrishnan, "EnergyEfficient Communication Protocol for wireless Micro sensor Networks," IEEE Proceedings of the Hawaii International Conference on System Sciences, January 2000, pp. 10.

[2] C. Intagagonwiwat, R. Govindan, and D. Estrin, "Directed Diffusion: A Salable and Robust Communication Paradigm for Sensor Networks," Mobicom'00, Boston, 2000, pp. 56-67.

[3] R. C. Shah and H. M. Rabaey, "Energy Aware Routing for Low Energy Ad Hoc Sensor Networks," presented at IEEE Wireless Communications and Networking Conference, 2002.

[4] D. Braginsky and D. Estrin. "Rumor Routing Algorithm for sensor Networks," in Proc. the First ACM International workshop on Wireless Sensor Networks \&Applications, Atlanta, September 2002, pp. 22-29.

[5] D. Ganesan, R. Govindan, S. Shenker, and D. Estrin. "Highly -Resilient, Energy-Efficient Multipath Routing in Wireless Sensor Networks," ACM Mobile Computing and Communications Review, vol. 5, no. 4, October 2001.

[6] I. F. Akyildiz, W. Su, Y. Sankarasubramaniam, and E. Cayirci, "Wireless Sensor Networks: A Survey," Computer Networks, March 2002.

[7] D. Ganesan, "Networking Issues in Wireless Sensor Networks," in Proc. the First ACM Conference on Embedded Networked Sensor Systems (SenSys)., 2003.

[8] Y. Xu, J. Heidemann, and D. Estrin, "Geography-informed energy conservation for adhoc routing," ACM MobiCom, pp. 70-84, 2001.

[9] Y. Yu, D. Estrin, and R. Govindan, "Geographical and energy aware routing: A recursive data dissemination algorithm for wireless sensor networks," UCLA-CSD, Technical Report TR- 01-0023, May 2001.

[10] C. Schurgers and M. B. Srivastava, "Energy efficient routing in wireless sensor networks," in Proc. MILCOM Communications for Network-Centric Operations: Creating the Information Force, McLean, VA, 2001.

[11] Z.-J. Wang, E. Bulut, and B. K. Szymanski, "Energy Efficient Collision Aware Multipath Routing for Wireless Sensor Networks".

[12] S. Lindsey et al., "Power-efficient gathering in sensorinformation systems," in IEEE Aerospace Conference Proceedings, vol. 3, pp. 1125-1130, 2002

[13] R. D. Poor. Gradient Routing in Ad Hoc Networks. [Online]. Available: http://www.media.mit.edu/pia/Research/ESP/texts/poorieeepaper.pdf

[14] D. B. Johnson and D. A. Maltz. "Dynamic Source Routing in Ad Hoc Wireless Networks. Mobile computing," Norwell, Mass: Kluwer Academic Publishers, 1996, pp. 153-181. 


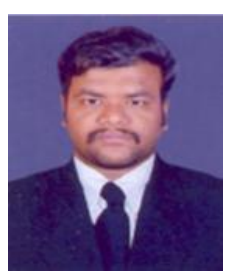

Kirankumar B. Balavalad was born on 8th November in the year 1985, and stays in Bagalkot city, Karnataka state, India. His educational background is he completed his BE from E\&C field in the year 2008 from Basaveshwar engineering college Bagalkot,

Karnataka state, India. And his master of technology in digital communication field from Basaveshwar engineering college, Bagalkot, in the year of 2010 . He is per suing his $\mathrm{PhD}$ in the area of MEMS.

$\mathrm{He}$ is currently working as assistant professor in department of E\&CE, Basaveshwar engineering college, Bagalkot, city-Bagalkot, state-Karnataka, country-India.

Prof Balavalad is life member of ISSS, UACEE, and member of IEEE.

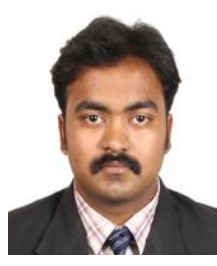

Ajayakumar C. Katageri was born on $21^{\text {st }}$ April in the year 1985, and stays in Bagalkot city, Karnataka state, India. His educational background is he completed his BE from E\&C field in the year 2008 from Basaveshwar engineering college Bagalkot, Karnataka state, India And his master of technology in digital communication field from Basaveshwar engineering college, Bagalkot, in the year of 2010 . He is

per suing his phd in the area of MEMS

$\mathrm{He}$ is currently working as assistant professor in department of E\&CE Basaveshwar engineering college, Bagalkot, city-Bagalkot, state-Karnataka, country-India.

Prof Katageri is life member of ISSS, UACEE, \& member of IEEE.

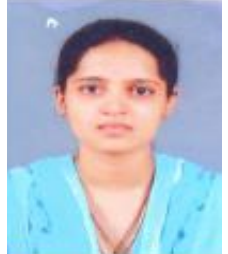

Poornima M. Chanal was born on 7th December in the year 1987, and stays in Bagalkot city, Karnataka state, India. Her educational background is she completed her BE from E\&C field in the year 2010 from Smt.Kamala and Shri Venkappa M Agadi college of engineering and Technology Laxmeshwar, Karnataka state, India. And she persuing master of technology in digital electronics and communication (Part time) field in Basaveshwar engineering college, Bagalkot.

She is currently working as Lecturer in department of E\&CE, Basaveshwar engineering college, Bagalkot, city -Bagalkot, stateKarnataka, country-India.

Prof Chanal is life member of ISTE.

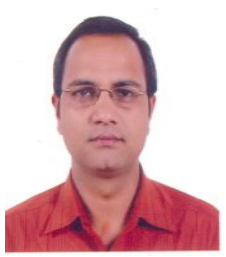

Gururaj S. Kori was born on 7th December in the year 1983, and stays in Bagalkot city, Karnataka state, India. His educational background is he completed his BE from E\&C field in the year 2008 from Basaveshwar engineering college Bagalkot, Karnataka state, India. And his presuing master of technology in digital communication field in Basaveshwar engineering college, Bagalkot. 